\title{
Curcumin nanoemulsion as a novel chemical for the treatment of acute and chronic toxoplasmosis in mice
}

This article was published in the following Dove Press journal:

International Journal of Nanomedicine

\section{Sanaz Jafarpour Azami ${ }^{1, *}$ \\ Aref Teimouri ${ }^{1,2, *}$ \\ Hossein Keshavarz' \\ Amir Amani ${ }^{3,4}$ \\ Fariba Esmaeili ${ }^{3}$ \\ Hamid Hasanpour ${ }^{1,5}$ \\ Samira Elikaee' \\ Hamid Salehiniya ${ }^{6}$ \\ Saeedeh Shojaee' \\ 'Department of Medical Parasitology and Mycology, School of Public Health, Tehran University of Medical Sciences, Tehran, Iran; ${ }^{2}$ Students Scientific Research Center, Tehran University of Medical Sciences, Tehran, Iran; ${ }^{3}$ Department of Medical Nanotechnology, School of Advanced Technologies in Medicine, Tehran University of Medical Sciences, Tehran, Iran; ${ }^{4}$ Natural Products and Medicinal Plants Research Center, North Khorasan University of Medical Sciences, Bojnurd, Iran; ${ }^{5}$ Department of Medical Parasitology and Mycology, School of Medicine, Ilam University of Medical Sciences, Ilam, Iran; \\ ${ }^{6}$ Department of Public Health, School of Health, Zabol University of Medical Sciences, Zabol, Iran}

*These authors contributed equally to this work

Correspondence: Saeedeh Shojaee Department of Medical Parasitology and Mycology, School of Public Health, Tehran University of Medical Sciences, Pour Sina St, Ghods St, Enghelab St, Tehran, Iran

Tel/fax+98 2l 8895 I392

Email shojaee1980@yahoo.com
Background: The aim of this study was to prepare curcumin nanoemulsion (CR-NE) to solve the problems associated with poor water solubility and low bioavailability of CR and to test its efficiency in the treatment of acute and chronic toxoplasmosis in mouse models.

Materials and methods: CR-NE $1 \%$ was prepared using spontaneous emulsification by soybean as oil phase; a mixture of Tween 80 and Tween 85 as surfactant; ethanol as cosurfactant and distilled water. Particle size and zeta potential of NE were assessed using Nano-ZS90 dynamic light scattering. Stability testing of NE was assessed after storage for 2 months at room temperature. In vivo experiments were carried out using $50 \mathrm{BALB} / \mathrm{c}$ mice inoculated with virulent RH strain (type I) and $50 \mathrm{BALB} / \mathrm{c}$ mice inoculated with avirulent Tehran strain (type II) of Toxoplasma gondii and treated with CR-NE (1\% w/v), CR suspension (CR-S, $1 \%$ w/v), and NE without CR (NE-no CR).

Results: The mean particle size and zeta potential of CR-NE included $215.66 \pm 16.8 \mathrm{~nm}$ and $-29.46 \pm 2.65 \mathrm{mV}$, respectively, and were stable in particle size after a three freeze-thaw cycle. In acute phase experiment, the survival time of mice infected with $\mathrm{RH}$ strain of $T$. gondii and treated with CR-NE extended from 8 to 10 days postinoculation. The differences were statistically significant between the survival time of mice in CR-NE-treated group compared with negative control group $(P<0.001)$. Furthermore, CR-NE significantly decreased the mean counts of peritoneum tachyzoites from 5,962.5 \pm 666 in negative control group to $627.5 \pm 73$ in CR-NE-treated mice $(P<0.001)$. Growth inhibition rates of tachyzoites in peritoneum of mice receiving CR-NE, CR-S, and NE-no CR included $90 \%, 21 \%$, and $11 \%$, respectively, compared with negative control group. In chronic phase experiment, the average number and size of tissue cysts significantly decreased to $17.2 \pm 15.6$ and $31.5 \pm 6.26 \mu \mathrm{m}$, respectively, in mice inoculated with bradyzoites of $T$. gondii Tehran strain and treated with CR-NE compared with that in negative control group $(P<0.001)$. Decrease of cyst numbers was verified by downregulation of BAG1 in treatment groups compared with negative control group with a minimum relative expression in CR-NE (1.12 \pm 0.28$)$, CR-S (11.76 \pm 0.87$)$, and NE-no CR (14.67 \pm 0.77$)$, respectively, $(P<0.001)$.

Conclusion: Results from the current study showed the potential of CR-S and CR-NE in treatment of acute and chronic toxoplasmosis in mouse models for the first time. However, CR-NE was more efficient than CR-S, and it seems that CR-NE has a potential formula for the treatment of acute and chronic toxoplasmosis, especially in those with latent bradyzoites in brain.

Keywords: curcumin nanoemulsion, curcumin suspension, Toxoplasma gondii RH strain, Toxoplasma gondii Tehran strain, soybean oil, blood-brain barrier, spontaneous emulsification

\section{Introduction}

Toxoplasma gondii, an obligate intracellular protozoan with worldwide distribution, can infect nearly all warm-blooded animals and humans. ${ }^{1}$ Humans are primarily infected by cc) and incorporate the Creative Commons Attribution - Non Commercial (unported, v3.0) License (http://creativecommons.org/licenses/by-nc/3.0/). By accessing the work you hereby accept the Terms. Non-commercial uses of the work are permitted without any further permission from Dove Medical Press Limited, provided the work is properly attributed. For permission for commercial use of this work, please see paragraphs 4.2 and 5 of our Terms (https://www.dovepress.com/terms.php). 
viable tissue cysts through the eating of raw or undercooked meats or by sporulated oocysts through ingestion of infected vegetables or water. Following ingestion, bradyzoites of cysts and sporozoites of oocysts are released and invade the epithelial cells of the intestine, where they are converted to tachyzoites. These tachyzoites can be disseminated to remote organs through the blood or lymphatic system and can induce acute and chronic infections. Vertical transmission of rapidly dividing tachyzoites from pregnant women to developing fetuses is another route of human infection. ${ }^{2}$ The acute acquired infection in immunocompetent patients is usually benign with self-limiting adenopathy. The chronic infection is asymptomatic in healthy people and associated with tissue-localized cysts, primarily in brain and muscle tissues. ${ }^{3}$ However, in AIDS and other immunocompromised patients, cysts may rupture and the bradyzoites revert to tachyzoites, causing acute-on-chronic toxoplasmosis. The acute-on-chronic toxoplasmosis may represent as toxoplasmic encephalitis, which may be fatal. ${ }^{4}$ Currently, recommended therapy for the treatment of toxoplasmosis includes a combination of pyrimethamine plus sulfadiazine. Although the available drugs include adequate efficiencies, they may include frequent toxic side effects as well. These side effects are described as allergy, bone marrow suppression, folic acid deficiency, and hematologic toxicity. ${ }^{5}$ Studies on medicinal plants include viable strategies for drug discovery. ${ }^{6}$ Curcumin (CR) is a natural phenolic compounds extracted from ground rhizomes of the perennial herb, Curcuma longa Linnaeus. The pharmacological safety and efficacy of CR make it a potential compound for the treatment and prevention of a wide variety of diseases such as chronic diseases, allergies, arthritis, wound healing, metal-induced liver damage, diabetes, migraine, Alzheimer's disease, and neurological disorders. $^{7-12}$ In addition to its harmless nature, CR provides a broad spectrum of biological and pharmacological properties, including antioxidant, antibacterial, anti-inflammatory, and anticarcinogenic properties. ${ }^{13-16}$ Furthermore, cytotoxic and parasiticidal properties of CR have been demonstrated in helminthic parasites such as Schistosoma mansoni, Schistosoma japonicum, and a wide range of protozoan parasites such as Leishmania, Giardia lamblia, Trypanosoma, and Plasmodium falciparum. ${ }^{17-23}$ Further properties include inhibitory activity against a Plasmodium berghei-infected model of malaria in mice. ${ }^{24}$ Despite numerous advantages of CR, it includes a number of inherent disadvantages such as low absorption and poor water solubility as well as low serum and tissue concentrations. ${ }^{25}$ The latter disadvantage decreases CR bioavailability (BA) ${ }^{25}$ In recent years, the use of nanomaterials as delivery systems and nutraceuticals have been introduced to medical societies as promising approaches to enhance the BA of therapeutic agents and bioactive compounds. ${ }^{26}$ Various formulations have been described to enhance the BA of CR. Of these formulations, nanoemulsion (NE) has extensively been developed to improve the solubility rate of CR. ${ }^{26} \mathrm{NEs}$, fine oil-in-water dispersions stabilized with small amounts of emulsifiers, are too small to scatter the light beams. Therefore, they seem clear instead of their optically opaque appearance. ${ }^{27}$ Up-to-date, only two "letters to editor" have described the effects of methanolic and ethanolic extracts of $C$. longa on tachyzoites of $T$. gondii; both reported a significant anti-Toxoplasma activity. ${ }^{28,29}$ To the best of the author's knowledge, no studies have been carried out on effects of CR-NE on acute and chronic toxoplasmosis. Therefore, the aim of this study was to prepare CR-NE using spontaneous emulsification method and to test its efficiency in the treatment of acute and chronic toxoplasmosis in mouse models.

\section{Materials and methods Compounds}

Soybean oil and CR were purchased from Sigma-Aldrich Co., St Louis, MO, USA. Polysorbate 80 and 85, ethanol, methanol, and chloroform were purchased from Merck KGaA, Darmstadt, Germany. Deionized distilled water (DW) was used in all experiments. The virulent RH strain (type I) and avirulent Tehran strain (type II) of $T$. gondii were provided by the Department of Medical Parasitology and Mycology, Tehran University of Medical Sciences, Tehran, Iran.

\section{Animals}

One hundred female BALB/c mice weighing 20-25 g were used in this study. Animals were housed under standard laboratory conditions (light-dark cycle conditions, controlled temperatures of $22^{\circ} \mathrm{C} \pm 2^{\circ} \mathrm{C}$ ) with ad libitum foods and fresh drinking water. Strategies were used to reduce pain and avoid animals suffering. The current study was approved by the Ethics Committee of Tehran University of Medical Sciences, Tehran, Iran (Approval No: 32426) on January 17, 2017. All animal procedures were carried out according to Guidelines for the Care and Use of Laboratory Animals published by the United States National Institutes of Health. These procedures were approved by the Ethical Committee of Tehran University of Medical Sciences, Tehran, Iran.

\section{Parasite strains}

Tachyzoites of $T$. gondii virulent RH strain (type I) and avirulent Tehran strain (type II) were passaged in BALB/c mice using intraperitoneal (IP) serial passages. ${ }^{30}$ Tachyzoites of $T$. gondii virulent RH strain (type I) were collected from 
peritoneal cavity of the infected mice, washed with PBS ( $\mathrm{pH}$ 7.4), and counted using hemocytometer slide. Infected mice with $T$. gondii Tehran strain (type II) were sacrificed after 2 months of infection, and their brains were removed and homogenized with $1 \mathrm{~mL}$ of PBS by passing through a 22-gauge needle. Brain cysts were counted by placing two drops of $20 \mu \mathrm{L}$ of the brain homogenates on slides and counting under a light microscope.${ }^{31}$ The number of cysts per brain was calculated using the following formula of number of counted cysts in two drops multiplied by 25, according to the World Organization for Animal Health. ${ }^{32}$

\section{Preparation of soybean oil of CR-NE}

Soybean oil was chosen as the oily phase, a mixture of Tween 80 and Tween 85 as the surfactants and ethanol as co-surfactant for NE preparation. The NE of CR was prepared using spontaneous emulsification method. Briefly, CR was dissolved in soybean oil by slowly addition of surfactant, co-surfactant, and DW using constant magnetic stirring at 100 rpm for 2 hours. ${ }^{33}$

\section{Characterization of the CR-NE}

Assessment of particle size and zeta potential of NE

Particle diameter of the NE was assessed using Nano-ZS90 dynamic light scattering (Malvern Instruments, Malvern, $\mathrm{UK}$ ) at a $90^{\circ}$ fixed angle at room temperature. D50 is the median, defined as the $50 \%$ of the particles below this value, similarly d 25 and $\mathrm{d} 75$ defined as the $25 \%$ and $75 \%$ of the particles below this value.

\section{Transmission electron microscopy (TEM) studies of CR-NE}

Morphology of the NE was visualized using Leo 906 E TEM (Carl Zeiss Meditec AG, Jena, Germany). NE was diluted with DW (1:4). A drop of the suspension was placed on a 200-mesh carbon-coated copper grid at room temperature. Grids containing the samples were dried extensively under a lamp, negatively stained using $2 \%$ uranyl acetate, and allowed to dry at room temperature. These grids were used for TEM study at $100 \mathrm{kV}$ using Leo $906 \mathrm{E}$ TEM and Gatan Digital Micrograph Software. ${ }^{34}$

\section{Stability of the NE}

Stability of the CR-NE was assessed by monitoring the change in phase separation, creaming, and discoloration after storage for 2 months at room temperature, and determination of particle size and zeta potential was investigated after a three freeze-thaw cycle. A formulation was kept in dry and dark place at room temperature.

\section{Treatment of acute toxoplasmosis}

Fifty 6- to 8-week-old female BALB/c mice were infected using IP inoculation of $1 \times 10^{4}$ tachyzoites of $T$. gondii RH strain and then equally divided into five groups containing 10 animals/group as follows: Group 1 included mice treated with $100 \mathrm{mg} / \mathrm{kg} / \mathrm{day}$ of CR-NE; Group 2 included mice treated with $100 \mathrm{mg} / \mathrm{kg} /$ day of CR-S; Group 3 included mice treated with NE-no CR; Group 4 included mice treated with $15 \mathrm{mg} / \mathrm{kg} /$ day of atovaquone as positive control group; and Group 5 included infected untreated mice as negative control group. ${ }^{35}$ Treatment started 4 hours postinoculation using oral gavage for 10 days with similar daily conditions. Drug doses were calculated based on the drug efficiency against T. gondii strains of the pilot studies (data not shown). Mice were monitored daily for 14 days, and mortality rate was documented for each group. To calculate the parasite load, peritoneal fluids of five mice from each group were collected at day 5 of treatment and tachyzoites were counted using light microscopy. Parasite growth was assessed by counting the number of tachyzoites in treatment and control groups using light microscopy at $400 \times$ magnification.

\section{Treatment of chronic toxoplasmosis}

Fifty female BALB/c mice were infected using IP inoculation of $25 \mathrm{~mL}$ of the mice brain suspension containing approximately 450 tissue cysts of $T$. gondii Tehran strain. The mice were equally divided into five subgroups as detailed in the acute phase study. Treatment was carried out for 30 days in 5 sequential weeks. Mice were weighed pre- and postexperiments. Behavioral and physical activities of the mice such as walking, jumping, standing, eating, body hair, and weight in treatment and control groups were monitored and recorded daily. After 2 months of infection, the animals were sacrificed and livers and spleens were removed and weighed. Furthermore, brains of the mice were removed and divided into two hemispheres under laminar flow tissue culture hood. One part was soaked in liquid nitrogen and stored at $-80^{\circ} \mathrm{C}$ for the study of BAG1 gene expression, and the other part was used for the calculation of tissue cysts. Squash smears were prepared from the mice brain tissues, and the number of cysts was counted and the size of cysts was calculated using light microscopy at $100 \times$ and $400 \times$ magnifications, respectively.

\section{Real-time PCR}

SYBR green-based real-time PCR (Qiagen Rotor-Gene Q, Hilden, Germany) was used to assess BAG1 expression in infected mice with $T$. gondii Tehran strain. The optimization of conditions for the SYBR green-based real-time PCR assay was 
performed in a $20 \mu \mathrm{L}$ reaction mixture that contained $10 \mu \mathrm{L}$ of RealQ Plus $2 \times$ Master Mix Green, $0.8 \mu \mathrm{L}(10 \mu \mathrm{M})$ of each primer, $7.4 \mu \mathrm{L}$ ddH2O, and $1.0 \mu \mathrm{L}$ synthetized cDNA. ${ }^{36}$

CFX96 ${ }^{\mathrm{TM}}$ Real-Time System (Bio-Rad Laboratories Inc., Hercules, CA, USA) was used for the amplification and detection. Amplification conditions were an initial step at $95^{\circ} \mathrm{C}$ for 2 minutes, followed by 40 cycles of $95^{\circ} \mathrm{C}$ for 15 seconds, $56.4^{\circ} \mathrm{C}$ for 20 seconds, and $72^{\circ} \mathrm{C}$ for 30 seconds. Melting curves were generated by monitoring the fluorescence of SYBR green signal from $65^{\circ} \mathrm{C}$ to $95^{\circ} \mathrm{C}$. Negative control contained $\mathrm{ddH} 2 \mathrm{O}$ and the PCV3 plasmid were used as positive control in the experiments. All reactions were conducted in triplicates.

\section{RNA extraction}

Total RNA was extracted from the mice brains as described previously. ${ }^{36}$ Briefly, $1 \mathrm{~mL}$ of the ice-cold RNX-plus solution (SinaClon BioScience, Tehran, Iran) was added to homogenized tissues samples and mixed by vortexing for 5-10 seconds. Then, $200 \mu \mathrm{L}$ of chloroform was added to the solution and centrifuged at $12,000 \times g$ for 15 minutes at $4^{\circ} \mathrm{C}$. An equal volume of isopropyl alcohol was added to the aqueous phase and centrifuged. Then, $1 \mathrm{~mL}$ of $75 \%$ ethanol was added to the supernatant and centrifuged. The RNA pellet was dissolved in a small volume of diethyl pyrocarbonate-treated water. RNA concentration was calculated using NanoDrop ${ }^{\circledR}$ ND-1000 UV-Vis spectrophotometer (NanoDrop Technologies, Wilmington, DE, USA) followed by denaturing gel electrophoresis on $1 \%$ agarose gels.

\section{cDNA synthesis and real-time PCR}

The cDNA was synthesized from total RNA using Prime Script RT-PCR Kit (Takara, Kusatsu, Japan), according to the manufacturer's instruction. Briefly, $4 \mu \mathrm{L}$ of $5 \times$ buffer, $1 \mu \mathrm{L}$ of oligo(dT) primer, $1 \mu \mathrm{L}$ of RT enzyme mix, $1 \mu \mathrm{L}$ of random hexamers, $10 \mu \mathrm{L}$ of the total extracted RNA, and $3 \mu \mathrm{L}$ of sterile DW were mixed in a sterile microtube to reach a total volume of $20 \mu \mathrm{L}$. The mixture was incubated in thermal cycler (Peqlab, Fareham, UK) for one cycle at $37^{\circ} \mathrm{C}$ for 15 minutes, one cycle at $85^{\circ} \mathrm{C}$ for 5 seconds, and one cycle at $4^{\circ} \mathrm{C}$ for 5 minutes. Furthermore, random samples without RT enzymes (no RT controls) were used to detect gDNA contamination in the samples. Primers of BAG1 (bradyzoite surface antigen 1) and GAPDH (glyceraldehyde 3-phosphate dehydrogenase) have been used for real-time PCR as reported in a previous study by the authors. ${ }^{36}$ Reaction conditions included a cycle at $50^{\circ} \mathrm{C}$ for 2 minutes, a cycle at $95^{\circ} \mathrm{C}$ for 15 minutes followed by 40 cycles at $95^{\circ} \mathrm{C}$ for 15 seconds, $60^{\circ} \mathrm{C}$ for 15 seconds, and $72^{\circ} \mathrm{C}$ for 15 seconds. A nontemplate control was used as negative control, and all reactions were carried out in triplicate to ensure reproducibility. The relative gene expression level for BAG1 in each sample was normalized by subtracting the cycle threshold (CT) of the housekeeping gene (GAPDH) to calculate the $\Delta \mathrm{CT} .{ }^{37}$

\section{Statistical analysis}

Statistical analysis was carried out using SPSS Software v.22 (IBM, Armonk, NY, USA). Statistical differences between the treatment and control groups were calculated using one-way ANOVA with CI of 95\%. Tukey's test was used as post hoc test for multiple comparisons. Dunnett's test was used to compare the control with the treatment groups. Moreover, Kaplan-Meier method was used for the comparison of survival rates between the studied groups in acute phases of toxoplasmosis. ${ }^{38} P$-values $\leq 0.05$ were considered statistically significant. Results were reported as mean $\pm \mathrm{SD}$.

\section{Results}

\section{Preparation of CR-NE}

In order to prepare soybean oil $\mathrm{NE}$ and encapsulate $1 \% \mathrm{CR}$, 10 and 40 batches were carried out, respectively. Finally, CR-NE was successfully prepared using CR ( $1 \% \mathrm{w} / \mathrm{v})$ in soybean oil (5\% w/v) as oily phase, mixture of Tween $80(24 \%)$ and Tween $85(12 \%)$ as surfactant concentrations, ethanol $(10 \%)$ as cosurfactant concentration and sterile DW (48\%).

\section{Characterization of CR-NE}

The mean particle size of CR-NE included $215.66 \pm 16.8 \mathrm{~nm}$ and the zeta potential included $-29.46 \pm 2.65 \mathrm{mV} . \mathrm{S} 0=\sqrt{\mathrm{d}} 75 /$ $\mathrm{d} 25$ was calculated for dispersion, which was $\cong 1.78$.

\section{Morphology of CR-NE}

The nanometer size of the prepared NE was assessed using TEM (Figure 1).

\section{Stability of CR-NE}

No significant changes were found in particle size and zeta potential after a three freeze-thaw cycle $(P=0.76$ and $P=0.86$, respectively) (Figure 2).

\section{Toxicity studies}

Oral toxicity studies were carried out on experimental infected mice with $T$. gondii Tehran strain. No clinical symptoms such as loss of activity, loss of body hair, and feeding and drinking disorders were found in mice within 


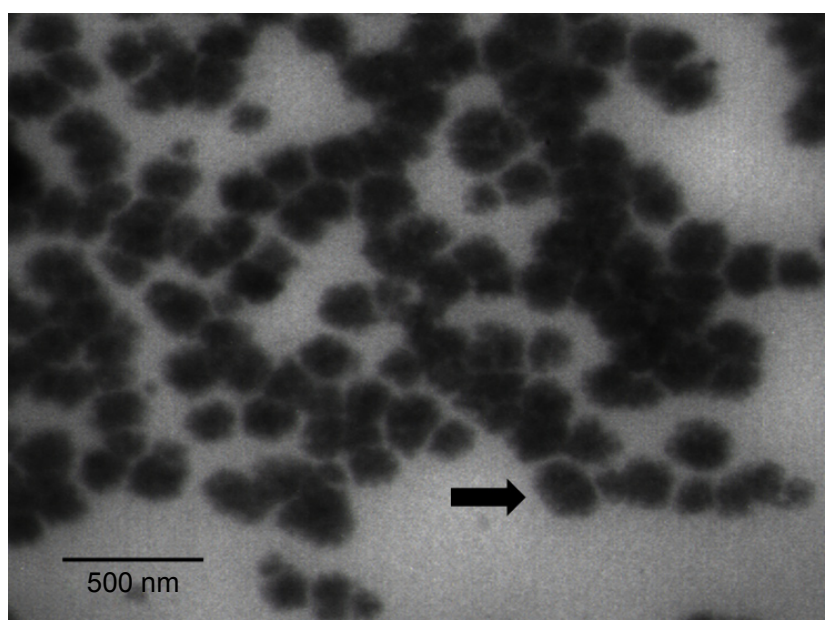

Figure I A TEM micrograph of CR-NE.

Notes: Arrow points at CR-NE.

Abbreviations: CR-NE, curcumin nanoemulsion; TEM, transmission electron microscopy.

the CR-NE-treated groups compared with those in control group. No significant differences were seen in mice body, liver, and spleen weights in both groups after 2 months ( $P$-values $>0.05)$ (Figure 3).
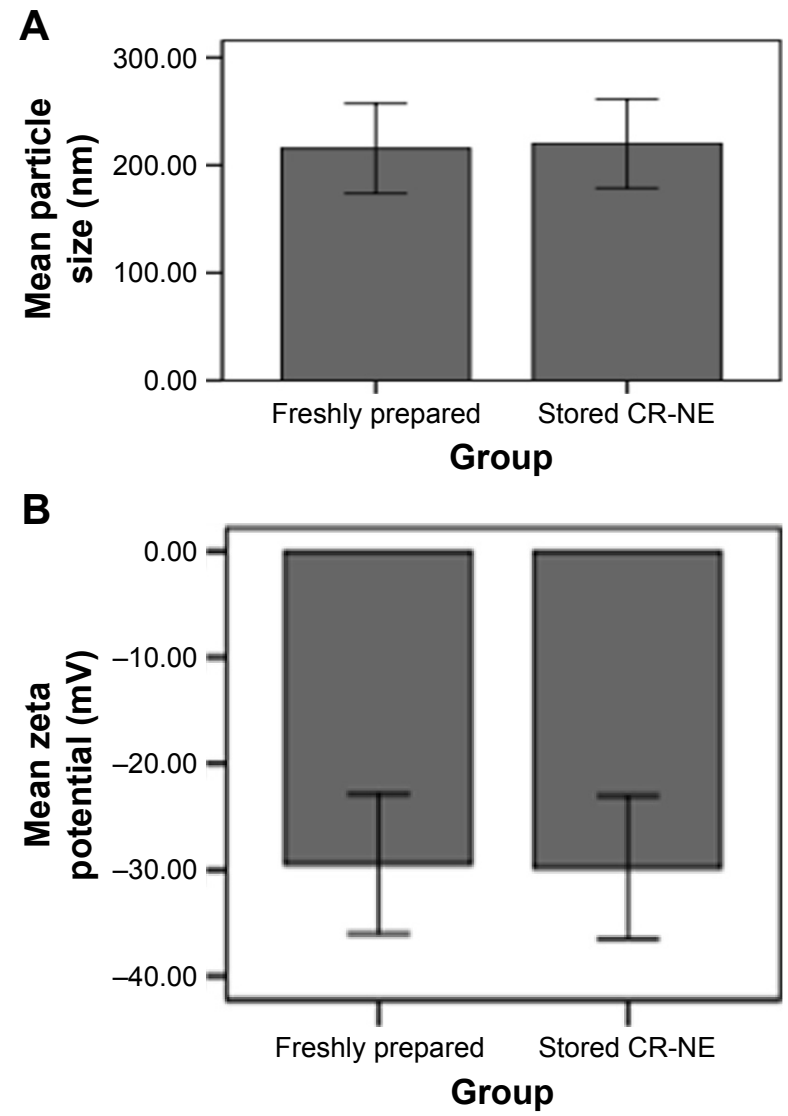

Figure 2 Effects of a 2-month storage of CR-NE on its particle size (A) and zeta potential (B) after a three freeze-thaw cycle.

Abbreviation: CR-NE, curcumin nanoemulsion.

\section{Acute experiments}

Mice survival time was assessed in treatment groups using Kaplan-Meier test (Figure 4). The mean survival time in all treatment groups was longer than that in negative control group (Table 1). In negative control group, infection symptoms were seen after day 4 and mice died within 5-7 days postinoculation. No symptoms of infection were observed in CR-NE-treated mice until day 6 of infection. In mice treated with CR-NE, the survival time extended from 8 to 10 days postinoculation (Table 1). Mice treated with CR-S and NE-no CR died 5-8 and 5-7 days postinoculation, respectively. Although survival time of mice was increased in all treatment groups compared with that in the negative control group, differences were statistically significant between the survival of mice treated with CR-NE compared with those of mice in negative control group $(P<0.001)$. Moreover, the survival of mice in CR-NE-treated group significantly increased compared with that in CR-S, NE-no $\mathrm{CR}$, and atovaquone-treated groups $(P<0.001$ and $P=0.004$, respectively). Results from one-way ANOVA analysis showed statistically significant differences between the mean counts of peritoneum tachyzoites in treatment groups at day 5 of inoculation compared with those in negative control group $(P<0.001)$. The differences were statistically significant between the mean counts of peritoneum tachyzoites in CR-NE-treated group compared with that in CR-S and NE-no CR-treated mice $(P<0.001)$. Growth inhibition rates of tachyzoites in mice receiving CR-NE, CR-S, and NE-no CR included $90 \%, 21 \%$, and $11 \%$, respectively, compared with those in negative control group. The positive control group represented $87 \%$ of the growth inhibition (Table 2).

\section{Chronic experiments}

The mean number and size of brain tissue cysts in treatment groups were lower than those of brain tissue cysts in negative control group (Figure 5). The average number and size of brain tissue cysts in CR-NE-treated mice included 17.2 \pm 15.6 and $31.5 \pm 6.26 \mu \mathrm{m}$, respectively. Number of brain tissue cysts significantly decreased in mice inoculated with bradyzoites of $T$. gondii Tehran strain and treated with CR-S and CR-NE compared with number of brain tissue cysts in negative control mice $(P<0.001)$. Significant differences were seen in size of cysts in CR-S and CR-NE-treated mice compared with size of cysts in negative control group $(P=0.02$ and $P<0.001$, respectively) (Figure 6). Furthermore, significant differences were found in number and size of brain tissue cysts in CRNE-treated mice compared with those in CR-S and NE-no CR-treated groups $(P<0.001)$. Moreover, number and size 


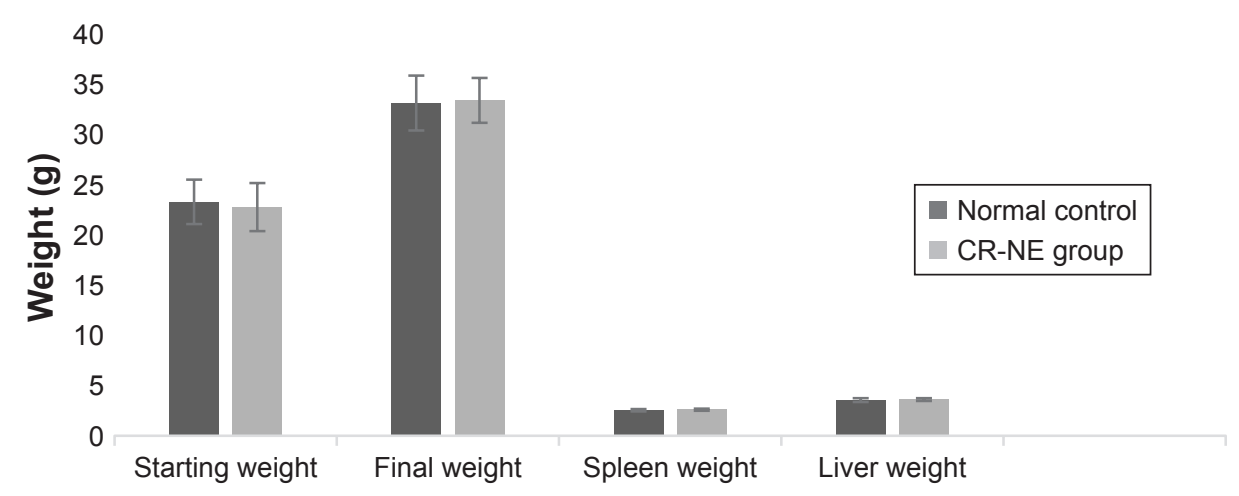

Figure 3 Toxicity studies including mice body, liver, and spleen weights in CR-NE-treated and control groups after 2 months. Abbreviation: CR-NE, curcumin nanoemulsion.

of brain tissue cysts significantly decreased in the positive control group compared with those in negative control mice $(P<0.001)$. However, no statistically significant differences were seen between the sizes of cysts in NE-no CR-treated mice compared with the sizes of cysts in negative control mice $(P=0.2)$.

\section{Gene expression}

The gene expression rate was assessed to verify efficiency of CR-NE on the decrease of tissue cysts in brains of infected mice with $T$. gondii Tehran strain. Agarose gel electrophoresis was used to assess RNA quality. Acceptable

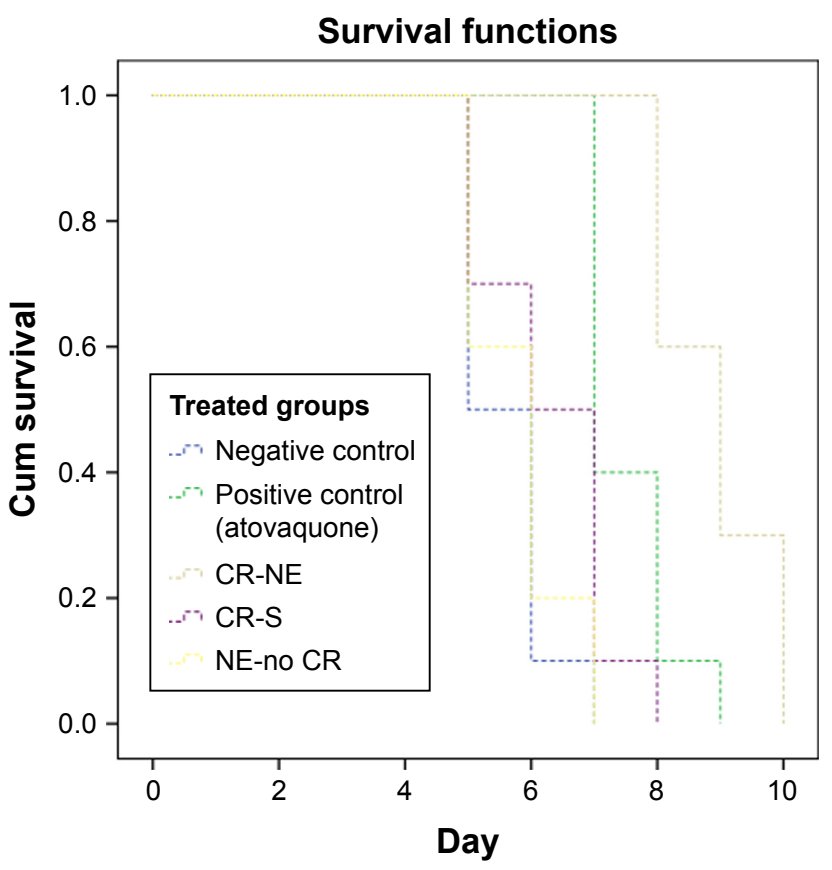

Figure 4 Survival rates of mice inoculated with tachyzoites of Toxoplasma gondii $\mathrm{RH}$ strain and treated with CR-NE, CR-S, and NE-no CR compared with those of mice in control groups $(n=10, P<0.001)$.

Abbreviations: CR-NE, curcumin nanoemulsion; CR-S, curcumin suspension; NE-no $C R$, nanoemulsion without curcumin. optical densities were seen at 1.8-2 with no gDNA contaminations. Downregulation of BAG1 was seen in treatment groups compared with that in negative control group with a minimum relative expression in CR-NE, CR-S, and NE-no CR (Figure 7). No significant differences were found in downregulation of BAG1 in NE-no CR compared with that in negative control group $(P=0.06)$. However, differences were statistically significant in downregulation of BAG1 in CR-NE and CR-S groups compared with that in negative control group $(P<0.001)$. Furthermore, significant differences were seen in downregulation of BAG1 in CRNE-treated mice compared with that in CR-S and NE-no CR-treated groups $(P<0.001)$.

\section{Discussion}

Results from the current study proved a good potential of $\mathrm{CR}$ in the treatment of acute and chronic toxoplasmosis in mouse models. Treatment of chronic toxoplasmosis is often challenging due to the poor reach of drugs to brain. The blood-brain barrier inhibits sufficient drug concentration in brain. Combined administration of sulfadiazine and pyrimethamine has demonstrated a good efficiency against acute toxoplasmosis but failed to show efficiency against chronic cerebral toxoplasmosis. ${ }^{39}$ Furthermore, prolonged use of these drugs may cause hematologic and renal toxicities. ${ }^{40}$ Other combined medicines include atovaquone and clindamycin, which are effective on acute infections and reduce severity of toxoplasmic encephalitis relapses. ${ }^{41,42}$ However, full therapeutic potential of these combinations is questioned by low BA, lack of sufficient brain penetration, and emerging resistance. ${ }^{43}$ High side effects and limited efficiencies of these drugs, especially against chronic toxoplasmosis, emerge further research to find safe and novel therapeutic agents. In the last decade, traditionally natural medicines have extensively been studied in drug discoveries because of their innate 
Table I Survival time of mice infected with Toxoplasma gondii RH strain tachyzoites and treated with CR-NE, CR-S, and NE-no CR compared with those of mice in control groups $(n=10, P<0.00 I)$

\begin{tabular}{|l|l|l|l|}
\hline \multirow{2}{*}{ Group } & Mean & \multicolumn{2}{l|}{} \\
\cline { 2 - 4 } & Mean survival time (days) \pm SD & $\mathbf{9 5 \%} \mathbf{C l}$ & Upper bound (days) \\
\cline { 3 - 4 } & & Lower bound (days) & 9.52 \\
\hline CR-NE & $8.9 \pm 0.87^{\mathrm{a}}$ & 6.1 & 7.05 \\
\hline CR-S & $6.3 \pm 1.01$ & 5.54 & 6.36 \\
\hline NE-no CR & $5.8 \pm 0.89$ & 5.24 & 6.1 \\
\hline Negative control & $5.6 \pm 0.69$ & 5.09 & 8.01 \\
\hline Positive control (atovaquone) & $7.5 \pm 0.71$ & 6.99 & \\
\hline
\end{tabular}

Notes: aCompared with negative control; $P \leq 0.05$.

Abbreviations: CR-NE, curcumin nanoemulsion; CR-S, curcumin suspension; NE-no CR, nanoemulsion without curcumin.

biodegradability and relatively safe nature for nontarget organisms ${ }^{44-48}$ One of these novel therapeutic medicines, CR, is a natural and nontoxic compound, which has been used for several centuries as a therapeutic and health-promoting agent. When administered as a dietary supplement, CR reduced amyloid $\beta$-peptide deposition in a transgenic mouse model demonstrating its ability to cross the blood-brain barrier in sufficient quantities to reduce amyloid plaque burden. ${ }^{49}$ Safety, tolerability, and nontoxicity of CR (even at high doses) are well verified using clinical trials..$^{50,51}$

Although the therapeutic use of Curcuma was first recorded as early as 1748 , the first article on the use of CR in human biliary disease was published in 1937 by Oppenheimer. ${ }^{52,53}$ After these initial introductions, interests in human studies on CR have increased dramatically. In therapeutic efficiency, results from the present study for the first time proved a good potential of CR-S and CR-NE in the treatment of acute and chronic toxoplasmosis in mouse models. Despite several studies on antibacterial, antiviral, and antifungal activities of $\mathrm{CR}$, no studies have been published on the effects of CR-NE against experimental toxoplasmosis. Results of acute experiments have shown that the mean survival time of mice in treatment group with CR-S and CR-NE is longer than that of mice in negative control group. However, differences in survival time of CR-NE-treated mice were statistically significant compared with those of mice in negative control group $(P<0.001)$. It is noteworthy that the mean survival time of mice in CR-NE-treated group was longer than that in atovaquone-treated group as positive control and these differences were statistically significant ( $P=0.004)$. Furthermore, results of this study have shown that CR-S and CR-NE significantly decreased the parasite load in peritoneal exudates of the treated mice infected with T. gondii RH strain compared with negative control mice $(P<0.001)$. Moreover, CR-S and CR-NE efficiencies were assessed against $T$. gondii Tehran strain in chronic experiments. Results of chronic experiment have shown that the average number and size of tissue cysts in CR-S and CR-NEtreated mice significantly decreased compared with those in negative control mice ( $P=0.02$ and $P<0.001$, respectively). In addition to microscopic studies for the analysis of number and size of brain cysts in mice, relative expression of the 30-kDa BAG1 protein has been assessed in mice with chronic T. gondii infection using real-time PCR. Results of decreased

Table 2 Anti-Toxoplasma activities of CR-NE, CR-S, and NE-no CR compared with control groups in peritoneal exudates of mice infected with $\mathrm{I} \times 10^{4}$ tachyzoites of Toxoplasma gondii $\mathrm{RH}$ strain

\begin{tabular}{|l|l|l|}
\hline Group & No of tachyzoites \pm SD $\left(\times 10^{4}\right)$ & $\%$ of growth inhibition $^{\text {a }}$ \\
\hline CR-NE & $627.5 \pm 73^{\mathrm{b}}$ & 90 \\
\hline CR-S & $4,745 \pm 340^{\mathrm{b}}$ & 21 \\
\hline NE-no CR & $5,362.5 \pm 430$ & $1 \mathrm{I}$ \\
\hline Positive control & $\mathrm{c}$ & 87 \\
\hline Negative control $^{\mathrm{d}}$ & $792.5 \pm 57^{\mathrm{b}}$ & - \\
\hline
\end{tabular}

Notes: aPercentage of the growth inhibition $=(I-$ No of tachyzoites after treatment/No of tachyzoites in negative control $) \times I 00$; b Indication of statistically significant differences compared with negative control $(P<0.001)$; 'Atovaquone; 'Without any treatment. Counts of tachyzoites were carried out in triplicate.

Abbreviations: CR-NE, curcumin nanoemulsion; CR-S, curcumin suspension; NE-no CR, nanoemulsion without curcumin. 


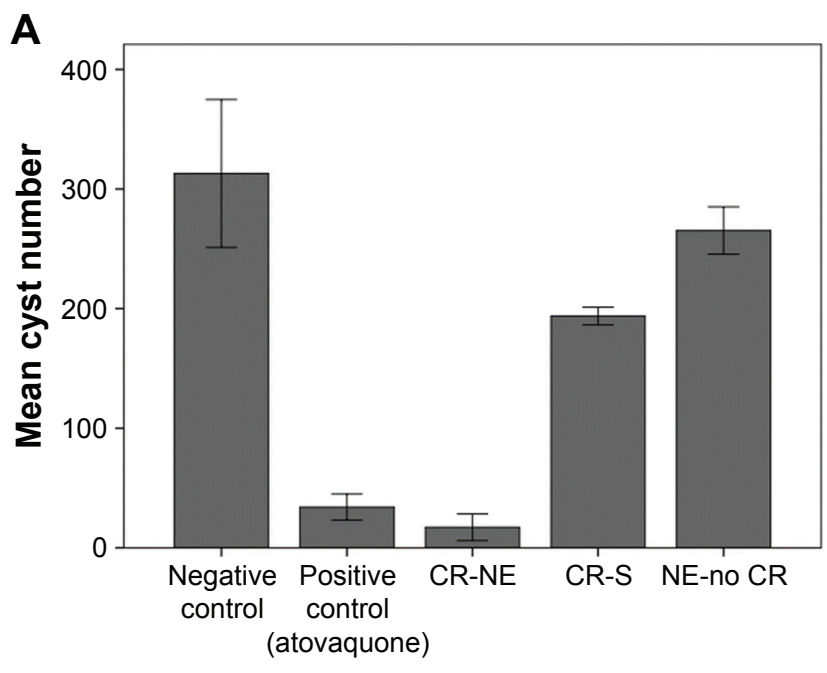

Treated groups

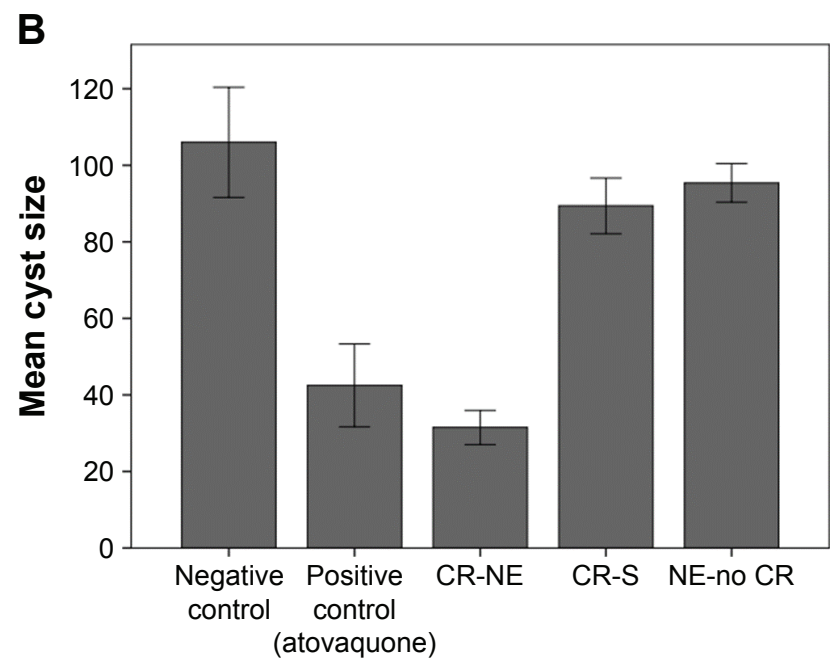

Treated groups

Figure 5 Mean \pm SD of the number $(\mathbf{A})$ and size $(\mathbf{B})$ of tissue cysts in brain of mice inoculated with bradyzoites of Toxoplasma gondii Tehran strain and treated with CR-NE, CR-S, and NE-no CR compared with those of brain tissue cysts in negative control mice $(n=10, P<0.00 I)$. Counts of tissue brain cyst and size of them measured in triplicate. Abbreviations: CR-NE, curcumin nanoemulsion; CR-S, curcumin suspension; NE-no CR, nanoemulsion without curcumin.

tissue cyst number and size in microscopic examination were verified using downregulation of the BAG1 gene.

Up-to-date, several studies have been carried out to assess the antimalarial activity of Curcuma extracts and CR and its derivatives. For example, in a study in Denmark, researchers isolated CR, demethoxycurcumin, and bisdemethoxycurcumin from rhizomes of $C$. longa and demonstrated that these components inhibited the parasite growth by $50 \%$ in vitro at 3-4.2 $\mu \mathrm{g} / \mathrm{mL}$ (approximately $8 \mu \mathrm{M}$ ) concentrations; almost 300 times greater than that required for the control drug of chloroquine. ${ }^{54}$ Similar studies showed that CR included an IC50 of $5 \mu \mathrm{M}$ against $P$. falciparum in vitro. Interestingly, they showed that $100 \mathrm{mg} / \mathrm{kg}$ of CR decreased blood parasitemia of $P$. berghei-infected mice by $>80 \%$ when administrated orally for 5 sequential days. ${ }^{55}$ In 2007 , Cui et al found that CR included IC50 ranging from 20 to $30 \mu \mathrm{M}$ against $P$. falciparum (both chloroquine-resistant and -sensitive laboratory strains). ${ }^{56}$ Similar findings were reported by other researchers. ${ }^{24}$ Moreover, efficiency of Curcuma extracts on protozoan parasites such as Babesia spp., Leishmania spp., Trypanosoma spp., Cryptosporidium parvum, and G. lamblia has already been proven. ${ }^{24}$ However, pharmacological mechanisms of $\mathrm{CR}$ are possibly associated with the compound inhibition of various biological cell signaling pathways and enzymes; molecular mechanisms of the CR parasiticidal activities need to be further investigated. ${ }^{57}$

In 1978, pilot studies by Wahlström and Blennow investigated the uptake of CR using Sprague Dawley rats. They
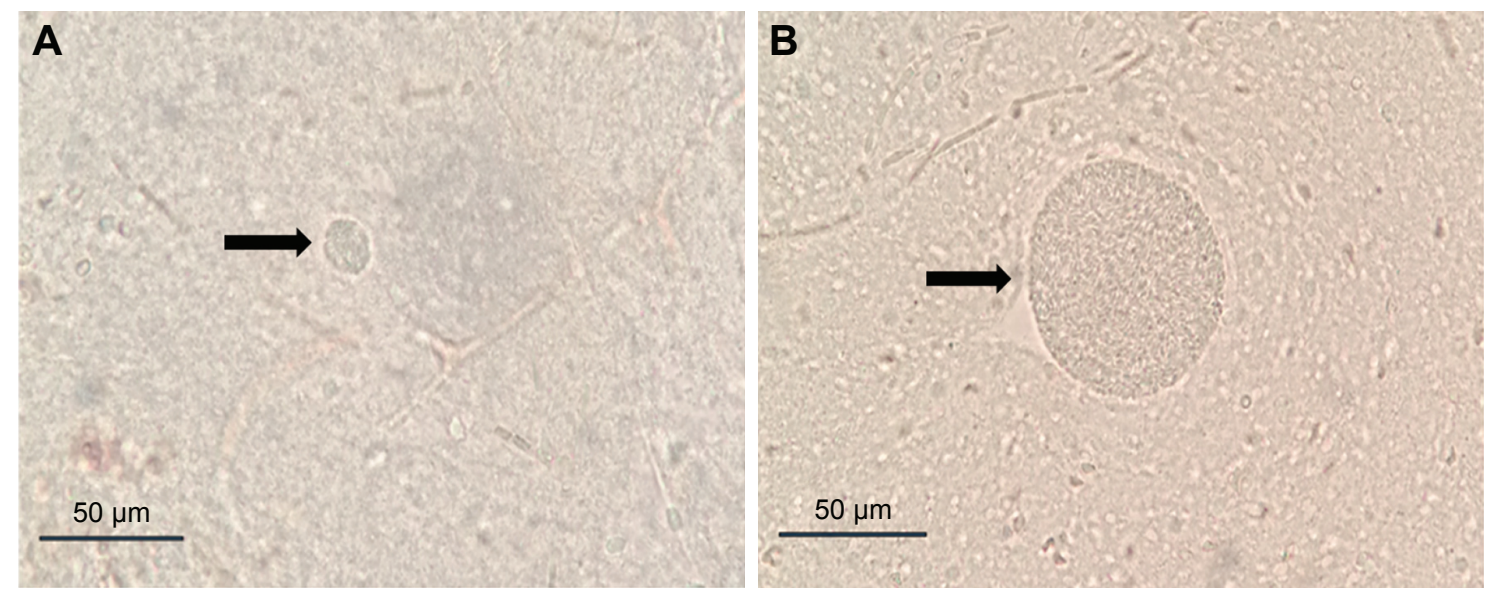

Figure 6 (A) Size of cysts in brains of mice treated with curcumin nanoemulsion; (B) size of cysts in brains of control mice without treatment. Notes: Arrow in $(\mathbf{A})$ and $(\mathbf{B})$ points at brain tissue cysts. 


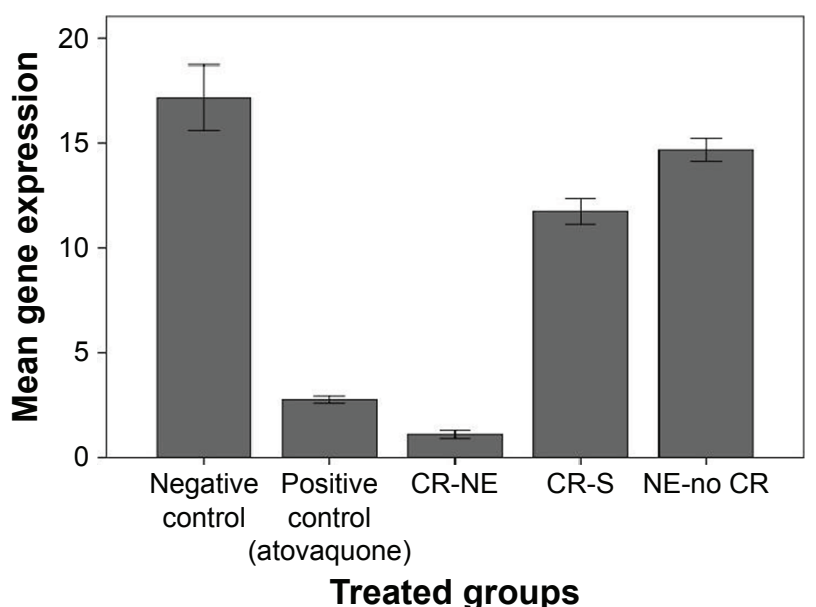

Figure 7 Mean expression \pm SD of BAGI, normalized with GAPDH as reference control in mice inoculated with bradyzoites of Toxoplasma gondii Tehran strain and treated with CR-NE, CR-S, and NE-no CR compared with that in mice in control group $(n=10, P<0.00 I)$. Experiments were carried out in triplicate.

Abbreviations: CR-NE, curcumin nanoemulsion; CR-S, curcumin suspension; NEno $C R$, nanoemulsion without curcumin.

reported that negligible quantities of $\mathrm{CR}$ were detected in blood plasma after oral administration of $1 \mathrm{~g} / \mathrm{kg}$ of CR in rats, suggesting that $\mathrm{CR}$ was poorly absorbed from the gut. ${ }^{58}$ However, inadequate oral BA of CR makes this potent drug ineffective. Similar to the natural form of CR, its nanoform is able to prevent and eliminate growth of microbes such as bacteria, fungi, and parasites. Bhawana et al showed improved suppressive roles of the aqueous dispersion of nanocurcumin compared with roles of the CR natural form against Aspergillus niger, Bacillus subtilis, Escherichia coli, Pseudomonas aeruginosa, Penicillium notatum, and Staphylococcus aureus..$^{59}$ Dandekar et al prepared a CRcontaining hydrogel NPs using hydroxyl propyl methyl cellulose and polyvinylpyrrolidone and demonstrated the advanced in vivo efficiency of this gel over the CR, regarding control of the pathogenesis of malarial parasites. ${ }^{60}$ Similar results were reported by Akhtar et al showing that the oral delivery of CR-loaded chitosan NPs to mice infected with lethal strains of Plasmodium yoelii caused the surveillance of all mice. In contrast, native CR only displayed a one-third survival rate ${ }^{61}$ In the current study, in vivo experiments demonstrated that CR-NE played a better role than that CR-S did against $T$. gondii tachyzoites in acute and chronic studies. The anti-Toxoplasma effect of CR-NE was statistically significant in CR-NE treatment group compared with that in other groups in acute and chronic experiments. This could occur due to improved BA and tissue distribution and therapeutic activity of CR-NE compared with native CR. The therapeutic effectiveness of CR formulation mainly depends on the enhanced availability of the drug in target organs. This, by itself, depends on adequate aqueous solubility of $\mathrm{CR}$, which results in an improvement in the drug BA. Compared with micron-sized emulsions, NEs provide greater surface areas including the potential of increased solubility. This potential exists because of combined large interfacial adsorption of the core compound, enhanced BA due to the rapid delivery of active compounds to cell membranes, as well as improved controlled release of the drug. ${ }^{62}$ Because of their small sizes, NEs do not cream after relatively long periods of storage and hence remain stable for longer times. ${ }^{63}$

According to several studies, the mean size of curcuminoid $\mathrm{NE}$ is often $>100 \mathrm{~nm} .{ }^{64}$ In the current study, the average particle size and zeta potential of the prepared CR-NE included $215.66 \mathrm{~nm}$ and $-29.46 \mathrm{mV}$, respectively. Furthermore, a high stability of the current CR-NE was observed over a storage period of 2 months at room temperature. No significant changes were found in particle sizes and zeta potentials after a three freeze-thaw cycle ( $P=0.76$ and $P=0.86$, respectively). This stability can be linked to high negative zeta potential of the current CR-NE. High positive or negative zeta potentials have been shown to contribute greatly to the stability of microemulsions and NEs due to the highly charged surfaces, which resist droplet aggregation. However, this is based on experiments and is not the unique indicator of predicted $\mathrm{NE}$ stability. ${ }^{65}$ Conventional emulsions usually show low stability as demonstrated by sedimentation of stored CR at room temperature. NEs often result in better physical stabilities. ${ }^{66}$ However, CR does not contact with the water in the external phase as it is incorporated in oily phases. Therefore, NEs likely provides inactive environments for $\mathrm{CR}$. In general, $\mathrm{CR}$ in NEs is effectively protected from degradation. ${ }^{66} \mathrm{An}$ advantageous substance used in preparation of the current CR-NE was soybean oil. Soybean is widely cultured due to nutritional benefits. Furthermore, it is one of the major protein and oil sources worldwide, especially in developing and underdeveloped countries. Antioxidant, anticancer, antibacterial, antiviral, and antifungal effects are listed as some properties of soybean. ${ }^{67-69}$ Therefore, soybean was chosen to prepare NEs in the present study. Benefits of the essential oils to decrease tissue cysts of $T$. gondii have been documented..$^{70}$ In this study, results from the acute-phase experiments revealed that the mean survival time in NE-no CR-treated mice was longer than that in negative control group. Moreover, the mean count of peritoneum tachyzoites in treatment mice was lower than that in control mice. In chronic experiments, decreased number and size of cysts in NE-no CR-treated mice in comparison with control mice possibly occurred due to antioxidant and antimicrobial nature of the soybean 
oil. Soybean seed contains various phytochemicals such as phenolic compounds, an excellent source of isoflavones. ${ }^{67}$ In fact, studies have reported that isoflavones of soybeans include daidzein, daidzin, genistein, genistin, and other phenolic compounds, which demonstrate strong antioxidant and anticarcinogenic properties. ${ }^{67}$ Therefore, these compounds can increase the product shelf life and promote beneficial effects on human health.

\section{Conclusion}

Results from the current study for the first time show the potential of CR-S and CR-NE in treatment of acute and chronic toxoplasmosis in mouse models. However, CR-NE was more efficient than CR-S in treatment of acute and chronic toxoplasmosis in mice. The current CR-NE includes a better mechanism of action than that atovaquone does as the available drug against $T$. gondii tachyzoites in acute and chronic cases. Therefore, CR-NE is emerged as a novel agent for the treatment of acute and chronic toxoplasmosis, especially in elimination of latent bradyzoites in brain. However, use of the CR nano therapeutics is still at early stages, and further studies are needed to investigate pharmacologic and pharmacokinetics of CR-NE. It is worth noticing that treatment of toxoplasmosis in immunocompromised patient is necessary; hence, further evaluation of CR-NE alone or combined with other nanoparticles against reactivated toxoplasmosis or interferon-gamma knockout murine models will be suggested.

\section{Acknowledgments}

We would like to acknowledge all staff of toxoplasmosis laboratory (Department of Medical Parasitology and Mycology, Tehran University of Medical Sciences, Tehran, Iran) for their useful collaboration. The authors would also like to express gratitude to Mahta Absalan for technical assistance.

\section{Disclosure}

The authors report no conflicts of interest in this work.

\section{References}

1. Dubey JP. Toxoplasmosis of Animals and Humans. 2nd ed. Boca Raton; New York:CRC Press; 2010:1-313.

2. Tenter AM, Heckeroth AR, Weiss LM. Toxoplasma gondii: from animals to humans. Int J Parasitol. 2000;30(12-13):1217-1258.

3. Montoya JG, Liesenfeld O. Toxoplasmosis. Lancet. 2004;363(9425): 1965-1976.

4. Tuazon CU. Toxoplasmosis in AIDS patients. J Antimicrob Chemother. 1989;23(Suppl A):77-82.

5. Martins-Duarte ES, Urbina JA, de Souza W, Vommaro RC. Antiproliferative activities of two novel quinuclidine inhibitors against Toxoplasma gondii tachyzoites in vitro. J Antimicrob Chemother. 2006;58(1):59-65.
6. Cowan MM. Plant products as antimicrobial agents. Clin Microbiol Rev. 1999;12(4):564-582.

7. Yallapu MM, Nagesh PK, Jaggi M, Chauhan SC. Therapeutic applications of curcumin nanoformulations. Aaps J. 2015;17(6):1341-1356.

8. Aggarwal BB, Sung B. Pharmacological basis for the role of curcumin in chronic diseases: an age-old spice with modern targets. Trends Pharmacol Sci. 2009;30(2):85-94.

9. García-Niño WR, Pedraza-Chaverrí J. Protective effect of curcumin against heavy metals-induced liver damage. Food Chem Toxicol. 2014; 69:182-201.

10. Bulboacă AE, Bolboacă SD, Stănescu IC, et al. The effect of intravenous administration of liposomal curcumin in addition to sumatriptan treatment in an experimental migraine model in rats. Int $J$ Nanomedicine. 2018;13:3093-3103.

11. Bulboacă A, Bolboacă DS, Suci S. Protective effect of curcumin in fructose-induced metabolic syndrome and in streptozotocin-induced diabetes in rats. Iran J Basic Med Sci. 2016;19(6):585-593.

12. Bulboacă AE, Bolboacă SD, Stănescu IC, Sfrângeu CA, Bulboacă AC. Preemptive analgesic and antioxidative effect of curcumin for experimental migraine. Biomed Res Int. 2017;2017:4754701-4754707.

13. Moghadamtousi SZ, Kadir HA, Hassandarvish P, Tajik H, Abubakar S, Zandi K. A review on antibacterial, antiviral, and antifungal activity of curcumin. Biomed Res Int. 2014;2014:186864.

14. Aggarwal BB, Harikumar KB. Potential therapeutic effects of curcumin, the anti-inflammatory agent, against neurodegenerative, cardiovascular, pulmonary, metabolic, autoimmune and neoplastic diseases. Int $J$ Biochem Cell Biol. 2009;41(1):40-59.

15. Chattopadhyay I, Biswas K, Bandyopadhyay U, Banerjee RK. Turmeric and curcumin: biological actions and medicinal applications. Curr Sci. 2004;87:44-53.

16. Feng T, Wei Y, Lee RJ, Zhao L. Liposomal curcumin and its application in cancer. Int J Nanomedicine. 2017;12:6027-6044.

17. de Moraes J. Natural products with antischistosomal activity. Future Med Chem. 2015;7(6):801-820.

18. Araújo CC, Leon LL. Biological activities of Curcuma longa L. Mem Inst Oswaldo Cruz. 2001;96(5):723-728.

19. Koide T, Nose M, Ogihara Y, Yabu Y, Ohta N. Leishmanicidal effect of curcumin in vitro. Biol Pharm Bull. 2002;25(1):131-133.

20. Das R, Roy A, Dutta N, Majumder HK. Reactive oxygen species and imbalance of calcium homeostasis contributes to curcumin induced programmed cell death in Leishmania donovani. Apoptosis. 2008;13(7):867-882.

21. Pérez-Arriaga L, Mendoza-Magaña ML, Cortés-Zárate R, et al. Cytotoxic effect of curcumin on Giardia lamblia trophozoites. Acta Trop. 2006;98(2):152-161.

22. Nose M, Koide T, Ogihara Y, Yabu Y, Ohta N. Trypanocidal effects of curcumin in vitro. Biol Pharm Bull. 1998;21(6):643-645.

23. Nagajyothi F, Zhao D, Weiss LM, Tanowitz HB. Curcumin treatment provides protection against Trypanosoma cruzi infection. Parasitol Res. 2012;110(6):2491-2499.

24. Haddad M, Sauvain M, Deharo E. Curcuma as a parasiticidal agent: a review. Planta Med. 2011;77(6):672-678.

25. Flora G, Gupta D, Tiwari A. Nanocurcumin: a promising therapeutic advancement over native curcumin. Crit Rev Ther Drug Carrier Syst. 2013;30(4):331-368.

26. Wang S, Su R, Nie S, et al. Application of nanotechnology in improving bioavailability and bioactivity of diet-derived phytochemicals. J Nutr Biochem. 2014;25(4):363-376.

27. Gupta A, Eral HB, Hatton TA, Doyle PS. Nanoemulsions: formation, properties and applications. Soft Matter. 2016;12(11):2826-2841.

28. Abdel-Hady NM,El-Sherbini GT, Morsy TA. Treatment of Toxoplasma gondii by two Egyptian herbs. J Egypt Soc Parasitol. 2008;38(3):1025-1026.

29. Al-Zanbagi NA, Zelai NT. Two methods for attenuating Toxoplasma gondii tachyzoites RH strain by using ethanol extract of Curcuma longa. J Egypt Soc Parasitol. 2008;38(3):965-976.

30. Teimouri A, Modarressi MH, Shojaee S, et al. Detection of toxoplasmaspecific immunoglobulin $\mathrm{G}$ in human sera: performance comparison of in house Dot-ELISA with ECLIA and ELISA. Eur J Clin Microbiol Infect Dis. 2018;37(8):1421-1429. 
31. Selseleh M, Modarressi MH, Mohebali M, et al. Real-time RT-PCR on SAG1 and BAG1 gene expression during stage conversion in immunosuppressed mice infected with Toxoplasma gondii Tehran strain. Korean J Parasitol. 2012;50(3):199-205.

32. Ole A. Manual of diagnostic tests and vaccines for terrestrial animals. Toxoplasmosis. 2008;1(6):1286.

33. Bouchemal K, Briançon S, Perrier E, Fessi H. Nano-emulsion formulation using spontaneous emulsification: solvent, oil and surfactant optimisation. Int J Pharm. 2004;280(1-2):241-251.

34. Naz Z, Ahmad FJ. Curcumin-loaded colloidal carrier system: formulation optimization, mechanistic insight, ex vivo and in vivo evaluation. Int J Nanomedicine. 2015;10:4293-4307.

35. Teimouri A, Azami SJ, Keshavarz H, et al. Anti-Toxoplasma activity of various molecular weights and concentrations of chitosan nanoparticles on tachyzoites of RH strain. Int J Nanomedicine. 2018;13 1341-1351.

36. Azami SJ, Amani A, Keshavarz H, et al. Nanoemulsion of atovaquone as a promising approach for treatment of acute and chronic toxoplasmosis Eur J Pharm Sci. 2018;117:138-146.

37. Livak KJ, Schmittgen TD. Analysis of relative gene expression data using real-time quantitative PCR and the 2(-Delta Delta C(T)) Method. Methods. 2001;25(4):402-408

38. Fletcher RH, Fletcher SW, Wagner EH. Clinical Epidemiology: The Essentials. 3rd ed. Baltimore: Williams and Wilkins; 1996.

39. Faucher B, Moreau J, Zaegel O, Franck J, Piarroux R. Failure of conventional treatment with pyrimethamine and sulfadiazine for secondary prophylaxis of cerebral toxoplasmosis in a patient with AIDS. J Antimicrob Chemother. 2011;66(7):1654-1656.

40. Crespo M, Quereda C, Pascual J, Rivera M, Clemente L, Cano T. Patterns of sulfadiazine acute nephrotoxicity. Clin Nephrol. 2000;54(1):68-72.

41. Djurković-Djaković O, Nikolić T, Robert-Gangneux F, Bobić B, Nikolić A. Synergistic effect of clindamycin and atovaquone in acute murine toxoplasmosis. Antimicrob Agents Chemother. 1999;43(9): 2240-2244.

42. Dunay IR, Heimesaat MM, Bushrab FN, et al. Atovaquone maintenance therapy prevents reactivation of toxoplasmic encephalitis in a murine model of reactivated toxoplasmosis. Antimicrob Agents Chemother. 2004;48(12):4848-4854

43. Hughes LM, Lanteri CA, O’Neil MT, Johnson JD, Gribble GW, Trumpower BL. Design of anti-parasitic and anti-fungal hydroxynaphthoquinones that are less susceptible to drug resistance. Mol Biochem Parasitol. 2011;177(1):12-19.

44. Lahlou M. The success of natural products in drug discovery. Pharmacol Pharm. 2013;4(3):17-31.

45. Yuan H, Ma Q, Ye L, Piao G. The traditional medicine and modern medicine from natural products. Molecules. 2016;21(5):559.

46. Song P, Zhang R, Wang X, He P, Tan L, Ma X. Dietary grape-seed procyanidins decreased postweaning diarrhea by modulating intestinal permeability and suppressing oxidative stress in rats. J Agric Food Chem. 2011;59(11):6227-6232.

47. Teimouri A, Haghi AM, Nateghpour M, et al. Antimalarial efficacy of low molecular weight chitosan against Plasmodium berghei infection in mice. J Vector Borne Dis. 2016;53(4):312-316.

48. Wang J, Han M, Zhang G, Qiao S, Li D, Ma X. The signal pathway of antibiotic alternatives on intestinal microbiota and immune function. Curr Protein Pept Sci. 2016;17(8):785-796.

49. Garcia-Alloza M, Borrelli LA, Rozkalne A, Hyman BT, Bacskai BJ. Curcumin labels amyloid pathology in vivo, disrupts existing plaques, and partially restores distorted neurites in an Alzheimer mouse model. J Neurochem. 2007;102(4):1095-1104.

50. Vogel A, Pelletier J. Examen chimique de la racine de Curcuma. [Chemical review of tuemric root]. J Pharm. 1815;1:289-300. French.
51. Gupta SC, Patchva S, Koh W, Aggarwal BB. Discovery of curcumin, a component of golden spice, and its miraculous biological activities. Clin Exp Pharmacol Physiol. 2012;39(3):283-299.

52. Loeber CC. De Curcuma officinarum. [The curcuma factories]. diss Inaug Halae. 1748. Latin.

53. Oppenheimer A. Turmeric (curcumin) in biliary diseases. Lancet. 1937;229(5924):619-621.

54. Rasmussen HB, Christensen SB, Kvist LP, Karazmi A. A simple and efficient separation of the curcumins, the antiprotozoal constituents of Curcuma longa. Planta Med. 2000;66(4):396-398.

55. Reddy RC, Vatsala PG, Keshamouni VG, Padmanaban G, Rangarajan PN. Curcumin for malaria therapy. Biochem Biophys Res Commun. 2005;326(2):472-474.

56. Cui L, Miao J, Cui L. Cytotoxic effect of curcumin on malaria parasite Plasmodium falciparum: inhibition of histone acetylation and generation of reactive oxygen species. Antimicrob Agents Chemother. 2007; 51(2):488-494.

57. Joe B, Vijaykumar M, Lokesh BR. Biological properties of curcumincellular and molecular mechanisms of action. Crit Rev Food Sci Nutr. 2004;44(2):97-111.

58. Wahlström B, Blennow G. A study on the fate of curcumin in the rat. Acta Pharmacol Toxicol. 1978;43(2):86-92.

59. Bhawana, Basniwal RK, Buttar HS, Jain VK, Jain N. Curcumin nanoparticles: preparation, characterization, and antimicrobial study. J Agric Food Chem. 2011;59(5):2056-2061.

60. Dandekar PP, Jain R, Patil S, et al. Curcumin-loaded hydrogel nanoparticles: application in anti-malarial therapy and toxicological evaluation. J Pharm Sci. 2010;99(12):4992-5010.

61. Akhtar F, Rizvi MM, Kar SK. Oral delivery of curcumin bound to chitosan nanoparticles cured Plasmodium yoelii infected mice. Biotechnol Adv. 2012;30(1):310-320.

62. Lovelyn C, Attama AA. Current state of nanoemulsions in drug delivery. J Biomater Nanobiotechnol. 2011;2(5):626-639.

63. Hernandez-Jaimes C, Fouconnier B, Perez-Alonso C, MunguiaGuillen JL, Vernon-Carter EJ. Degradation activity: formulation optimization, characterization, and stability of Equisetum arvense extract nanoemulsion. J Disper Sci Technol. 2013;34:64-71.

64. Lu PS, Inbaraj BS, Chen BH. Determination of oral bioavailability of curcuminoid dispersions and nanoemulsions prepared from Curcuma longa Linnaeus. J Sci Food Agric. 2018;98(1):51-63.

65. Park HJ, Kong M. Stability investigation of hyaluronic acid based nanoemulsion and its potential as transdermal carrier. Carbohydr Polym. 2011;83:1303-1310.

66. Rachmawati H, Budiputra DK, Mauludin R. Curcumin nanoemulsion for transdermal application: formulation and evaluation. Drug Dev Ind Pharm. 2015;41(4):560-566.

67. Villalobos MC, Serradilla MJ, Martín A, Ordiales E, Ruiz-Moyano S, Córdoba MG. Antioxidant and antimicrobial activity of natural phenolic extract from defatted soybean flour by-product for stone fruit postharvest application. J Sci Food Agric. 2016;96(6):2116-2124.

68. Nakashima H, Okubo K, Honda Y, Tamura T, Matsuda S, Yamamoto N. Inhibitory effect of glycosides like saponin from soybean on the infectivity of HIV in vitro. AIDS. 1989;3(10):655-658.

69. Naim M, Gestetner B, Zilkah S, Birk Y, Bondi A. Soybean isoflavones. Characterization, determination, and antifungal activity. J Agric Food Chem. 1974;22(5):806-810.

70. Dahbi A, Bellete B, Flori P, et al. The effect of essential oils from Thymus broussonetii Boiss on transmission of Toxoplasma gondii cysts in mice. Parasitol Res. 2010;107(1):55-58. 


\section{Publish your work in this journal}

The International Journal of Nanomedicine is an international, peerreviewed journal focusing on the application of nanotechnology in diagnostics, therapeutics, and drug delivery systems throughout the biomedical field. This journal is indexed on PubMed Central,

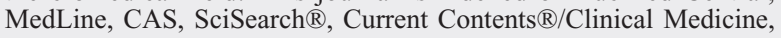

Journal Citation Reports/Science Edition, EMBase, Scopus and the Elsevier Bibliographic databases. The manuscript management system is completely online and includes a very quick and fair peer-review system, which is all easy to use. Visit http://www.dovepress.com/ testimonials.php to read real quotes from published authors.

Submit your manuscript here: http://www.dovepress.com/international-journal-of-nanomedicine-journal 